\title{
Studying local anisotropy properties of the most energetic cosmic rays by Monte Carlo clustering analysis
}

\author{
H. R. Marquez Falcon \\ Institute of Physics and Mathematics \\ Universidad Michoacana de San Nicolas de Hidalgo - UMSNH \\ Morelia, Mich., Mexico \\ E-mail: hugo@ifm.umich.mx

\section{M. Villasenor Cendejas} \\ Institute of Physics and Mathematics \\ Universidad Michoacana de San Nicolas de Hidalgo - UMSNH \\ Morelia, Mich., Mexico \\ E-mail: villasen@ifm.umich.mx
}

\begin{abstract}
We have used the binomial test to search for clusters of Auger events with energies greater than $60 \mathrm{EeV}$ that deviate in an statistically significant way from the null hypothesis of an isotropic distribution. We have found a set of 11 clusters that fail the test when we restrict to significances lower than 0.0014. The centroids of these clusters are prime candidates to be correlated with single high-intensity astrophysical sources, i.e., repeaters, or regions of the sky with a high concentration of potential sources.
\end{abstract}

4th School on Cosmic Rays and Astrophysics,

August 25-September 04, 2010

Sao Paulo Brazil 


\section{Introduction}

The search for the sources of the most energetic cosmic rays was one of the main motivations to construct the Pierre Auger Observatory. Since 2004, the Auger Observatory has collected a large sample of events with energies greater than the GZK cutoff [1]. The energy spectrum of these events has been used to establish a suppression of the flux of cosmic rays above $4 \times 10^{19} \mathrm{eV}$ [2]. The arrival directions of these events have been used to establish a correlation with the positions of AGNs $[3,4]$ located within a distance of $75 \mathrm{Mpc}$ from the Earth.

We used the events with energies greater than $60 \mathrm{EeV}$ to search for local anisotropies, i.e., points in the sky that show the highest statistically significant deviations from isotropy. We used the binomial test [5] to quantify the significances of these deviations, i.e., we estimate the probabilities that the data events are grouped in the observed 2-fold, 3-fold, 4-fold, 5-fold and 6-fold clusters as a consequence of random deviations from an assumed isotropic distribution, i.e., we took a fully isotropic distribution at all energy scales as our null hypothesis.

We searched for clusters of events with multiplicities in the 2-6 range that deviate maximally from the null hypothesis. We found 11 clusters that failed the test when we restrict to significances lower than 0.0014. All these clusters are confined in cones with half-cone angles ranging from $1.12^{\circ}$ to $2.88^{\circ}$. These results are statistical conclusions based on our present data in a way independent of catalogues of candidate sources.

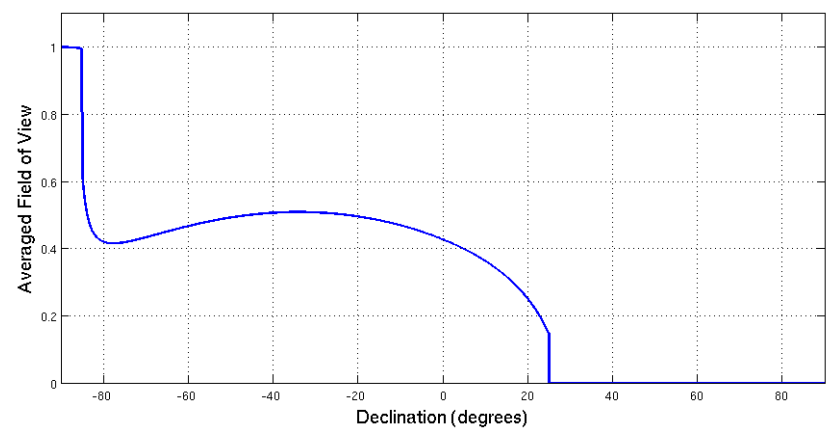

Figure 1: Field of view of the Auger Observatory in equatorial coordinates. The instantaneous field of view within $60^{\circ}$ around the zenith was evaluated at a single latitude of $-35^{\circ}$ and it was averaged over a full sidereal day. The average field of view varies only with the declination coordinate as shown on this plot.

\section{Description of the statistical method}

We use the binomial distribution [5] to calculate the statistical significance of the clusters of events observed in the arrival directions of the most energetic cosmic rays detected by the Auger Observatory up to the end of 2009 [6]. We assume an isotropic distribution as our null hypothesis; it is important to stress that isotropy is assumed for all energy scales. The binomial test is well suited for small samples and it incorporates in a natural way the dependence of the significance on the energy of the events.

Therefore, given a number of events $N_{E}$ observed with energies greater than $E$ over the whole sky, the significance $S$ is defined as the probability that $N_{o b s}$ or more events are observed inside a 
cone with a half-cone angle equal to $\delta$. This significance represents the probability that the given cluster results from a fluctuation of an isotropic distribution of the observed $N_{E}$ events with energies greater than $E$. The significance can be calculated from the following formula:

$$
S(\delta, E)=1-\sum_{k=0}^{N_{o b s}-1} \frac{N_{E} !}{k !\left(N_{E}-k\right) !} p_{\delta}^{k}\left(1-p_{\delta}\right)^{N_{E}-k}
$$

where $p_{\delta}$ is the probability that a single event occurs inside the cone, under the same isotropic conditions. We evaluate the value of $p_{\delta}$ by direct integration of the averaged field of view of the Auger Observatory, see Fig. 1, over the cone "surface".

We apply the statistical test to look for clusters of events, with specific multiplicities ranging from 2 to 6, occurring in the data set with significant deviations from isotropy for all the events with energies greater than $60 \mathrm{EeV}$.

The specific procedure we used to find event clusters is the following:

1. Find all clusters of a given multiplicity in the range 2-6 among all the Auger events with $E>60 \mathrm{EeV}$.

2. Calculate the center of gravity of the cluster and the angle $\delta_{o}$ from the centroid to the farthest event. If this angle is lower than 1.2 degrees we directly assigned this bottom value since this is the angular resolution of the Auger Observatory [7].

3. Calculate the significance by using Eq. 2.1 with $E$ equal to the minimum energy of the events in the cluster, $E_{\text {min }}$.

4. Sort the clusters in increasing order of $S$.

5. Remove clusters with at least one event in common starting from the top to the bottom of the above list, i.e., we systematically prioritize low values of S.

6. Keep only the clusters that have values lower than $0.0014=S_{\text {background }}$. This cutoff in the significance has been establish simulating isotropic events and this is explained in the next topic.

\section{Sackground}

For a given set of detected events there are so many combinations of 2-fold, 3-fold, 4-fold, 5fold and 6-fold clusters however only a few are significant. In order to establish what is significant or not we proceed to generate 1000 sets of 69 isotropic events (number of events collected by the Auger Observatory up to the end of 2009 with energy grater than $60 \mathrm{EeV} \mathrm{[6]),} \mathrm{in} \mathrm{each} \mathrm{case} \mathrm{it} \mathrm{was}$ calculated all significances of all doublets choosing only the ten most significant values in each case and filling with them a histogram. The $S_{\text {background }}=0.0014$ was set from the mean value of the distribution of significances, see Fig. 2. 


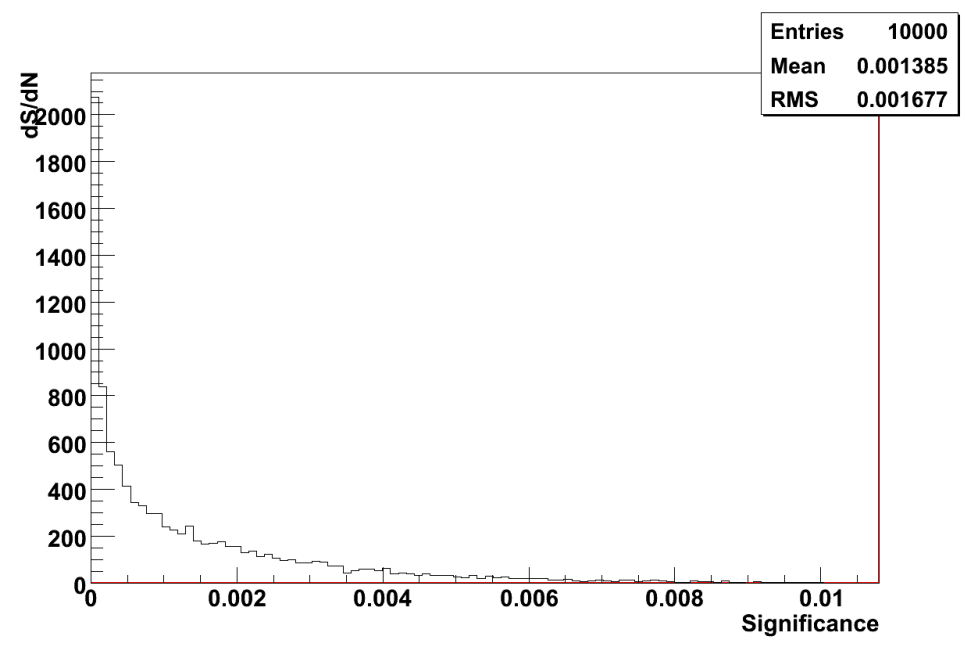

Figure 2: Histogram filled with the significances of simulating 1000 sets of 69 isotropic events where in each set only the ten lowest significances are chosen.

\begin{tabular}{|c|c|c|c|}
\hline Index & $\operatorname{RA}\left({ }^{o}\right), \operatorname{Dec}\left({ }^{o}\right)$ & $\delta_{o}\left({ }^{o}\right)$ & $\mathrm{S}$ \\
\hline \hline 1 & $201.7,-34$ & 1.88635 & 0.000411853 \\
\hline 2 & $268.15,-60.75$ & 1.2 & $5.60966 \mathrm{e}-05$ \\
\hline 3 & $222.5,-43.55$ & 1.58838 & 0.000202929 \\
\hline 4 & $331.45,0.85$ & 2.06518 & 0.000410284 \\
\hline 5 & $53.35,-6$ & 1.83334 & 0.000292187 \\
\hline 6 & $201.5,-55.1$ & 1.2 & $5.9177 \mathrm{e}-05$ \\
\hline 7 & $145.2,-18.3$ & 1.89881 & 0.000389664 \\
\hline 8 & $218.65,-52.65$ & 1.23667 & $7.00929 \mathrm{e}-05$ \\
\hline 9 & $310.8,-54.35$ & 2.56958 & 0.00125356 \\
\hline 10 & $80.55,-15.9$ & 2.16618 & 0.000648332 \\
\hline 11 & $200.067,-45.7$ & 2.87624 & $4.47676 \mathrm{e}-05$ \\
\hline
\end{tabular}

Table 1: List of the clusters with significances lower than $0.0014=S_{\text {background }}$ found according to the procedure described in the method. The coordinates of the centroids are given in equatorial coordinates.

\section{Results and discussion}

We found 11 centroids of cluster events (Table 1) with significances lower than $0.0014=$ $S_{\text {background }}$ with half-cone angles ranging from $1.12^{\circ}$ to $2.88^{\circ}$. These points have high probability in the sky to search for sources in their near neighborhoods. The last centroid in the list (index 11) matches with the position of Centaurus A and also is the most significant. Moreover this method by itself associates 3 events to CenA and split out the centroids 2 and 6 (Figure 3) as separate sources despite they are very close, this is the most important conclusion of applying the method to the Auger ultra-energetic events. 


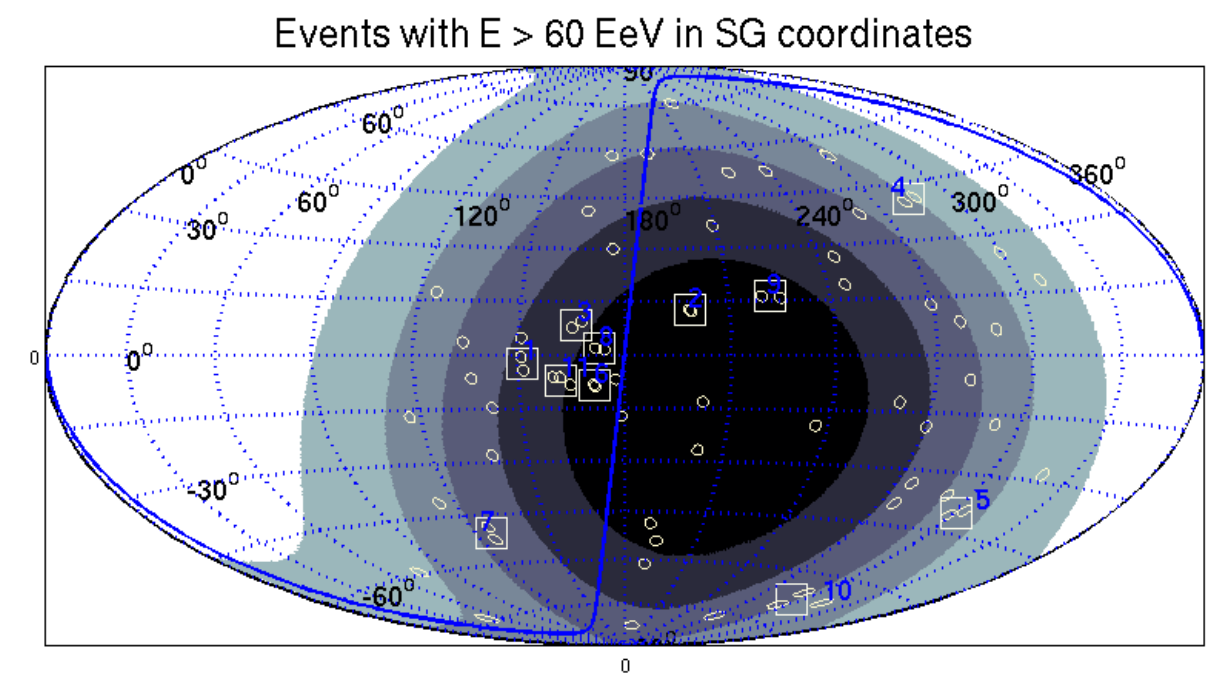

Figure 3: The arrival directions of the Auger events that possess energies above $60 \mathrm{EeV}$ are represented by the lemon circles in SG coordinates using an Aitoff projection of the sky. The blue solid line represents the Galactic plane. The squares are centered at the positions of the centroids generated by the method. The five colored bands correspond to equal-exposure areas.

\section{References}

[1] Greisen, K., Phys. Rev. Lett., 16 (1966) 748. Greisen, K., Phys. Rev. Lett., 16 (1966); Zatsepin, G. T., Kuzmin, V. A., PisIma Zh. Eksp. Teor. Fiz, 4 (1966) 114.

[2] [Pierre Auger Collaboration], Phys. Rev. Lett. 101, 061101 (2008).

[3] Pierre Auger Collaboration, Science 318, 938 (2007).

[4] Pierre Auger Collaboration, Astropart.Phys.29:188-204,2008, Erratum-ibid.30:45,2008.

[5] See any book on statistics, e.g., E. Keyszig, Introductory Mathematical Statistics: Principles and Methods. John Wiley and Sons, Inc, 1970

[6] The Pierre Auger Collaboration, Sep 2010, arXiv:1009.1855v1 [astro-ph.HE].

[7] The Pierre Auger Collaboration, Jan 2009, arXiv:0901.3138v1 [astro-ph.HE]. 